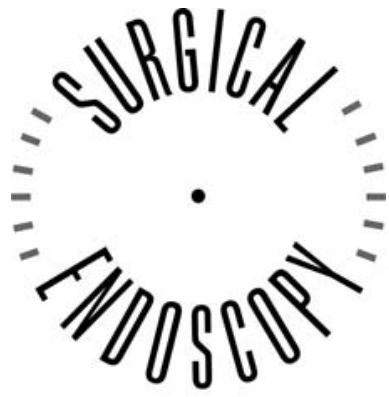

and Other Interventional Techniques

\title{
Medical liability insurance claims on entry-related complications in laparoscopy
}

\author{
Jan Wind, ${ }^{1}$ Jan E. L. Cremers, ${ }^{2}$ Mark I. van Berge Henegouwen, ${ }^{1}$ Dirk J. Gouma, ${ }^{1}$ Frank-Willem Jansen, ${ }^{3}$ \\ Willem A. Bemelman ${ }^{1}$ \\ ${ }^{1}$ Department of Surgery, Academic Medical Centre, Amsterdam, the Netherlands \\ 2 MediRisk, Utrecht, the Netherlands \\ ${ }^{3}$ Department of Gynaecology, Leiden University Medical Centre, Leiden, the Netherlands
}

Received: 5 December 2006/Accepted: 3 January 2007/Online publication: 5 April 2007

\begin{abstract}
Background: Installation of the pneumoperitoneum is an essential part of laparoscopic surgery. Creation can be performed by either the open or a closed technique. The aim of this study was to assess the number of and contributing factors to entry-related complications in medical liability insurance claims in the Netherlands.

Methods: A retrospective chart review was performed, including all malpractice claims filed at MediRisk, which is presently the largest medical liability mutual insurance company for institutions, mainly hospitals, in healthcare in the Netherlands.

Results: From January 1993 to December 2005, 41 claims were identified as entry-related complications which comprised $18 \%$ of all laparoscopy-related complications leading to claims. Most were young (median age $=35$ years) female patients who had routine, nonadvanced, laparoscopic procedures planned as shortstay or day-care procedures. The claims were equally divided between general surgery $(n=20)$ and gynecology $(n=21)$. A total of 51 structures were injured. There were 18 vascular structure injuries, 30 bowel injuries, and three other injuries. An open entry technique was used in only two (5\%) patients. Vascular injury was exclusively associated with closed entry. In only $19(46 \%)$ patients the entry-related complication was diagnosed peroperatively, consisting of $70 \%$ of the vascular and $25 \%$ of the bowel injuries. Twenty-six patients (64\%) were admitted to the intensive care unit for a median of five days. There was no mortality. Besides conversion, the majority of the patients filed a claim to compensate for a longer hospital stay and related costs.
\end{abstract}

Presented at the 14th International Congress of the European Association for Endoscopic Surgery (EAES), core scientific program, Berlin, Germany, 13-16 September 2006

Correspondence to: Willem A. Bemelman
A payment was made in $17(57 \%)$ of the 30 settled claims.

Conclusions: Medical liability claims concerning laparoscopic entry-related complications comprised a fifth of all laparoscopy-related claims. Claims concerning entry-related complications occurred in young patients who had routine, nonadvanced procedures. In the investigated cases most claims involved the closed-entry technique.

Key words: Laparoscopy - Entry technique - Pneumoperitoneum - Trocar - Open - Closed - Veress needle - Bowel injury — Vascular injury

Despite the ongoing technological advances in laparoscopic surgery, the creation of a pneumoperitoneum and the additional introduction of surgical instruments remain a potential dangerous first step that can result in serious injuries to the viscera and major intra- and retroperitoneal vessels. The reported incidence of vascular and bowel injuries varies and is between approximately 0.05 and 0.5 complication per 100 laparoscopic procedures [1-4]. Although the incidence of entry-related complications is rare, the result of major vascular and unrecognized bowel injuries is serious, often leading to severe morbidity and even mortality. The overall mortality rate is reported to be about $4 \%$, increasing to $21 \%$ for unrecognized bowel injury [5-7]. In several reports the fatalities result from about $75 \%$ vascular injuries and the remaining $25 \%$ from unrecognized bowel injuries $[4,5,8]$. Furthermore, it has been calculated that one half of all laparoscopic complications can be blamed on the entry technique [9-11].

In general, there are two techniques to establish the pneumoperitoneum and to enter the abdominal cavity. The first is the closed (blind) entry, which is performed by using a Veress needle to establish the pneumoperi- 
toneum followed by trocar insertion or, less frequently, direct trocar insertion without previously establishing a pneumoperitoneum. The second method is the open technique in which a small laparotomy is performed. Successively, skin, rectus fascia, and peritoneum are incised under direct vision, followed by blunt (Hasson's) trocar insertion. Subsequently, the pneumoperitoneum is created [1, 12]. With respect to the prevention of entry-related complications, neither of these two techniques is supported by solid evidence and there is an ongoing debate, mainly between gynecologists who favor the closed-entry technique and surgeons who favor the open-entry technique, about which technique is better [1, 10]. Many general surgeons suggest that the open-entry technique results in the same number of visceral lesions as does the closed-entry technique, but significantly fewer vascular lesions [1, 13]. Furthermore, it could be hypothesized that another advantage of open entry is that at least part of a bowel injury is immediately seen under direct vision. Because of the lack of evidence, the European Association for Endoscopic Surgery (EAES) practical clinical guideline on the pneumoperitoneum could not state which of the entry techniques is preferred [9].

To answer this question with a high level of evidence, a randomized comparison of the open and closed access techniques with a sample size of over 200,000 patients would be required to detect a reduction of major complications from 1 per 1000 to 0.5 per 1000 [14]. Therefore, it is important to continue to report on this topic because one has to rely on accumulating evidence of a lower level. In this study medical liability data sources were used to provide evidence on entry-related injuries.

The aim of this study was to assess the number of entry-related complications that provoked medical liability insurance claims for laparoscopic surgery at the largest medical liability mutual insurance company for institutions in healthcare in the Netherlands. Furthermore, the used entry technique (i.e., open vs. closed), distribution of injured organs, and predictive factors for litigation were assessed.

\section{Patients and methods}

\section{Data source}

A retrospective chart review was performed which included all malpractice claims filed at MediRisk concerning entry-related complications from January 1993 to December 2005. MediRisk was founded in 1993 and is presently the largest medical liability mutual insurance company for institutions, mainly hospitals, in the healthcare industry in the Netherlands. In 1993 MediRisk insured 21 hospitals. At the end of 2005 the number of insured hospitals included 80 of the 101 Dutch hospitals (Fig. 1). The insured institutes are broadly representative of the Dutch teaching and nonteaching hospitals, with the exception of all eight academic hospitals in the Netherlands, none of which are insured at MediRisk.

\section{Definitions and inclusion and exclusion criteria}

An entry-related complication was defined as a direct injury related to the insertion of either the Veress needle or the first trocar, including preperitoneal insufflations and injuries to epigastric vessels, intraperi- toneal viscera and vessels, and retroperitoneal viscera and vessels. Port-site hernias were excluded from this analysis.

Claims in which the etiology of the injury was not clear and causes other than insertion (e.g., coagulation injury or other procedure-related complications) that could not be ruled out based on operative reports and/or histologic examination were excluded. Furthermore, injuries caused by second-trocar insertion were also excluded.

\section{Claim characteristics}

All included claims were reviewed and the following data were extracted using a preformatted sheet: age, gender, comorbidity, prior abdominal surgery and/or previous intra-abdominal infectious events, body mass index (BMI, $\mathrm{kg} / \mathrm{m}^{2}$ ), specialty (i.e., gynecology or general surgery), urgency, experience (i.e., resident or consultant), informed consent, indication for surgery, entry technique (i.e., open or closed), type of entry-related complication, moment of diagnosis of the entryrelated complication, postoperative recovery including data such as reoperations, intensive care unit (ICU) admission, and postoperative morbidity and mortality. The entry-related complications were classified as "bowel," "vascular," or "other." The gastrointestinal tract was divided into three parts: stomach, small intestine, and large intestine. Vascular injuries were divided into retroperitoneal, intraperitoneal, and abdominal wall (epigastric vessels). Furthermore, the vascular injuries were subdivided into arterial, venous, or vascular not specified.

Data were calculated as median values with ranges for continuous and discrete data, unless otherwise specified. Categorical data are presented as frequencies or percentages. Analysis was done using the SPSS v12.0 package (SPSS Inc., Chicago, IL, USA).

\section{Results}

Filed claims concerning laparoscopic procedures and entry-related complications

In the study period a total of 10,552 claims were filed at MediRisk (Fig. 1) of which 229 (2\%) involved gynecologic and surgical laparoscopic procedures. Of these 229 claims, 50 were identified as entry-related complications. Subsequently, seven claims were excluded because the etiology of the injury was not clear and causes other than Veress needle or trocar insertion could not be ruled out. Furthermore, two claims were excluded because the injury was caused by insertion of the second trocar. The final analysis comprised 41 claims, which is $18 \%$ of all laparoscopy-related claims filed at MediRisk. Figure 2 shows the relationship between all laparoscopy-related claims and the number of entry-related complications. The yearly number of filed claims concerning entry-related complications remained stable during the study period.

\section{Characteristics of the entry-related complications}

The median age of the 41 patients included in this study was 35 years (range $=14-81$ ). Median BMI was 24.0 $\mathrm{kg} / \mathrm{m}^{2}$ (range $=18.6-55.9 \mathrm{~kg} / \mathrm{m}^{2}$ ). Sixteen $(39 \%) \mathrm{pa}-$ tients had a high BMI $\left(>25.0 \mathrm{~kg} / \mathrm{m}^{2}\right)$ and six $(15 \%)$ had a low BMI $\left(<20 \mathrm{~kg} / \mathrm{m}^{2}\right)$. Twenty-one patients $(51 \%)$ had a history of abdominal surgery (1-3 procedures), with the ratio between general surgery and gynecology 20:21. The surgical patients consisted of $17(85 \%) \mathrm{fe}-$ males and $3(15 \%)$ males. Residents were involved in six $(15 \%)$ claims. In both surgical and gynecologic patients, 
Table 1. Planned operative procedures in the 41 patients with an entry-related complication

\begin{tabular}{ll}
\hline Planned procedure & Number of patients $(n=41)$ \\
\hline Gynecology & \\
Sterilization & 11 \\
Diagnostic laparoscopy & 4 \\
Ovarian cystectomy & 2 \\
Adhesiolysis & 2 \\
Ectopic pregnancy & 1 \\
Adnex extirpation & 1 \\
Surgery & $10^{\mathrm{a}}$ \\
Cholecystectomy & 3 \\
Diagnostic laparoscopy & 3 \\
Appendectomy & 2 \\
Hernia repair & 1 \\
Gastric banding & 1 \\
Gastric perforation &
\end{tabular}

${ }^{\text {a }}$ Including two open-entry techniques; in all other patients a closedentry technique was used

the planned procedures were routine and nonadvanced and in $37(90 \%)$ patients they were elective (Table 1).

A total of 51 structures were injured (Table 2). There were 18 vascular structure injuries consisting mainly of retroperitoneal vessels $(n=13,72 \%)$ of arterial origin ( $n=10,77 \%), 30$ bowel injuries, and 3 other injuries. Thirty-six patients had one injury and $5(12 \%)$ patients had a combination of entry-related complications (range $2-3)$. The injured retroperitoneal arteries included the right iliac artery $(n=2,20 \%)$, the left iliac artery $(n=2,20 \%)$, and the abdominal aorta $(n=3,30 \%)$; in the remaining $3(30 \%)$ cases the injured artery was not reported. The large-bowel injuries comprised mainly the transverse $(n=4,40 \%)$ and left-side colon $(n=4$, $40 \%)$. Two (5\%) patients had an open entry-related complication. Both patients had a history of a previous laparotomy. In one patient the exact type of entry technique was not mentioned. A closed-entry technique was used in the remaining $38(93 \%)$ patients. All vascular entry-related complications were seen after the closed-entry technique. In $15(39 \%)$ cases in which the closed-entry technique was used, it could not be determined whether the Veress needle or the primary trocar was responsible for the injury. In the remaining closedentry cases, the Veress needle was responsible in 12 (32\%) and the first trocar in 11 (29\%). In only five of the latter cases was the design of the trocar was reported: four shielded trocars and one optical trocar.

Thirteen (32\%) claims provided additional narrative comments by the surgeon about the cause of the injury, citing various contributing factors. These comments included adhesions $(n=4)$, "overshoot" with the trocar $(n=2)$, distended intestine $(n=2)$, very thin patient $(n=2)$, pregnancy $(n=1)$, postpartum $(n=1)$, obesity $(n=1)$, and device problems during introduction $(n=1)$.

\section{Time of diagnosis and postoperative course}

In $19(46 \%)$ cases the entry-related complication was diagnosed peroperatively resulting in 16 conversions. In the remaining $22(54 \%)$ cases the injury was diagnosed at
Table 2. Type of entry-related complications $(n=51)$ in the 41 patients included in this study

\begin{tabular}{lll}
\hline $\begin{array}{l}\text { Type of entry-related } \\
\text { complication }\end{array}$ & $\begin{array}{l}\text { Gynecology } \\
(n=21)\end{array}$ & $\begin{array}{l}\text { Surgery } \\
(n=20)\end{array}$ \\
\hline $\begin{array}{l}\text { Vascular } \\
\text { Epigastric vessels }\end{array}$ & 1 & 1 \\
$\quad$ Retroperitoneal vessels & 4 & 9 \\
$\quad$ Intraperitoneal vessels & \\
Bowel & 2 & 1 \\
$\quad$ Stomach & 1 & 1 \\
$\quad$ Small intestine & 9 & $9^{\mathrm{c}}$ \\
$\quad$ Large intestine & 6 & $4^{\mathrm{c}}$ \\
Other & & $2^{\mathrm{b}}$ \\
$\quad$ Uterus & 0 & 0 \\
$\quad$ Preperitoneal insufflation & 1 & \\
\hline
\end{tabular}

${ }^{a}$ Mesenterial artery or omental artery

b One patient was 22 weeks pregnant; ${ }^{\mathrm{c}}$ including one open-entry technique

Table 3. Time of diagnosis (no delay vs. delayed diagnosis) and postoperative course of the 41 patients included in this study

\begin{tabular}{|c|c|c|}
\hline & $\begin{array}{l}\text { No delay } \\
(n=19)\end{array}$ & $\begin{array}{l}\text { Delayed diagnosis } \\
(n=22)\end{array}$ \\
\hline \multicolumn{3}{|l|}{ ICU stay } \\
\hline Number of patients (\%) & $8(42 \%)$ & $18(82 \%)$ \\
\hline Median days (range) & $1(1-18)$ & $8(1-60)$ \\
\hline $\begin{array}{l}\text { Patients with postoperative } \\
\text { morbidity }(\%)\end{array}$ & $6(32 \%)$ & $17(77 \%)$ \\
\hline Permanent harm & $5(26 \%)$ & $4(18 \%)$ \\
\hline
\end{tabular}

$\mathrm{ICU}=$ intensive care unit

median postoperative day 2 (range $=0-5$ ), resulting in one or more reoperations and a complicated postoperative course (Table 3). Eighty percent of the combined injuries and $70 \%$ of the vascular injuries were diagnosed peroperatively; the remaining vascular and combined injuries were diagnosed on the same day. Only $25 \%$ of the bowel injuries were diagnosed peroperatively; the remainder were diagnosed at median postoperative day 2. Twenty-six patients $(64 \%)$ were admitted to the ICU for a median of 5 days (range $=1-60$ ). Seventy percent of the patients with a vascular injury were admitted to the ICU for a median of 2 days (range $=1-34$ ). Fiftyeight percent of patients with a bowel injury were admitted to the ICU for a median of 8 days (range $=1-$ $60)$. There were no claims for fatal injuries. Nine (22\%) patients suffered permanent injury ( $>1$ year). The latter consisted of post-traumatic stress disorder $(n=3)$, neuropathy $(n=2)$, postventilation lung damage $(n=1)$, premature childbirth due to uterus perforation $(n=1)$, permanent enterocuteneous fistula $(n=1)$, and intermittent claudication $(n=1)$.

From patient records we determined that informed consent was properly established, i.e., documented and understood by the patient, in only 13 (32\%) claims. In the remaining claims, informed consent was not documented or not given at all. In $48 \%$ of the claims, complications such as bowel injury or the chance of conversion to laparotomy were discussed preoperatively. According to the records a postoperative meeting between surgeon and patient, in which the complications 


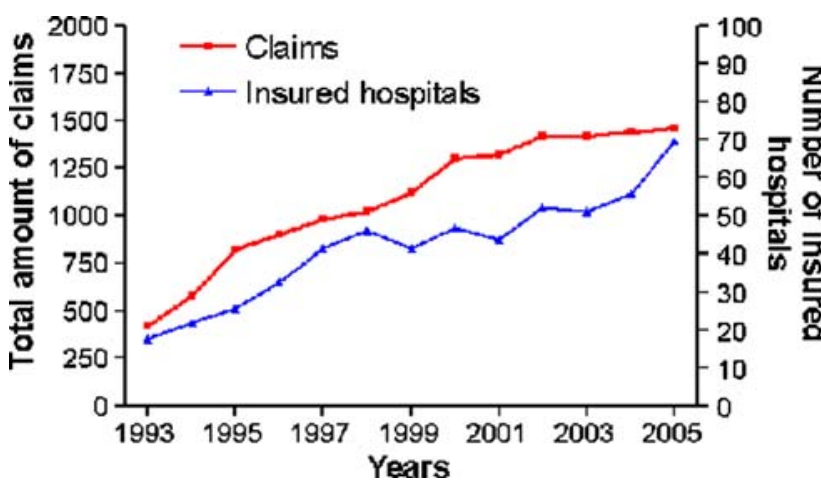

Fig. 1. The number of hospitals insured and the amount of claims that were filed between 1993 and 2005.

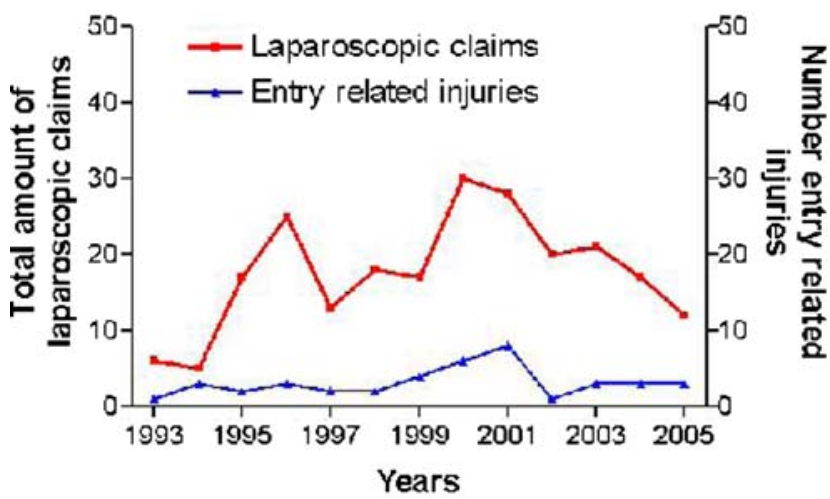

Fig. 2. The relation between Laparoscopic related claims in total and entry related injuries between 1993 and 2005.

that occurred were discussed, had taken place in only 16 (39\%) cases.

The majority of the patients claimed financial compensation for the prolonged hospital stay, related costs such as traveling expenses and domestic care, and the midline laparotomy scar. Thirty (73\%) claims were settled at the end of the study period and a payment was made in $17(57 \%)$ of the settled claims.

\section{Discussion}

Several randomized controlled trials and meta-analyses have demonstrated that laparoscopic surgery is superior to laparotomy in terms of morbidity, postoperative recovery, and length of hospital stay [15, 16]. Having achieved broad acceptance, minimally invasive surgery is a fast-expanding surgical discipline. Nevertheless, the initiation of laparoscopy, i.e., the creation of pneumoperitoneum followed by the introduction of the surgical instruments remains a potentially dangerous first step, which is exclusively associated with the laparoscopic approach.

The present study demonstrates that medical liability claims involving entry-related injuries comprised about one fifth of all laparoscopic surgery-related claims filed at MediRisk. The claims were equally distributed between general surgery and gynecologic procedures.
However, this distribution does not imply that the incidences of entry-related complications are comparable because the number of laparoscopic procedures of both specialities performed during the study period is not known.

In both the general surgery and gynecology cases the planned procedure was generally a routine one and elective in nature. Furthermore, these procedures involved mostly young female patients who planned to be operated on in a day-care setting or in short-stay surgery. The consequences of an entry-related complication in these young patients who underwent a routine procedure is striking, however. The entry-related complication resulted in one or more laparotomies, stay in the ICU, and prolonged hospitalization. Furthermore, 22\% of the patients suffered from permanent injury.

Since the first reports on entry-related complications, many articles have been published on this subject and trocars have been introduced with new design features, including retractable shields and optical trocars to allow direct viewing during insertion $[5,17]$. Furthermore, to avoid entry-related complications it is important to identify patients at risk, e.g., those with adhesions from previous laparotomy and obese and very thin patients. However, despite this identification, entry-related complications still occur at a constant rate $[1,2,8]$. In a recent survey of the U.S. Food and Drug Administration (FDA) Fuller et al., who reviewed all reports from January 1997 to June 2002, identified 31 fatal and 1353 nonfatal trocar injuries. Most fatalities involved vascular injuries [5].

In our study most claims were provoked by injuries caused by the closed-entry technique, and vascular injury was exclusively caused by the closed-entry technique. However, this does not mean that the incidence of entry-related complications for the closed-entry technique is much higher compared with that of the openentry technique because the numbers of performed closed- and open-entry techniques are unclear. However, retroperitoneal vascular injury is associated with "overshoot" of the introduction of the Veress needle or the first trocar. Theoretically, this can be avoided by using an open introduction. It is known that an open introduction might reduce the incidence of this serious complication. On the other hand, bowel injuries are not fully avoided by the open-entry technique.

Historically, gynecologists have been trained in the closed-entry technique. Although the technique of open laparoscopy was first described by the gynecologist Hasson in 1971 [12], only a few gynecologists use the open-entry technique $[1,18]$. Some have reported comparable or even higher complication rates with the openentry technique in gynecologic case series [1]. However, in these studies gynecologists did not use the open-entry technique frequently; it was used mainly in selected patients who had prior abdominal surgery. Consequently, these patients already were at a higher risk for entry-related complications. This explains why in a systematic review by Merlin et al. [19] the risk of major complications initially appeared to be higher for the open-entry technique. When only prospective series were taken into account the opposite was shown, a rel- 
ative risk of $0.30(95 \% \mathrm{CI}=0.09-1.03)$ in favor of the open-entry technique. It was noted that retrospective studies compared a high-risk with a low-risk patient population, while the prospective studies investigated an unselected patient population [19]. Furthermore, in a Japanese survey of laparoscopic surgeons, Hashizume et al. [13] reported that during the study period $96.6 \%$ of the surgeons changed their method of establishing a pneumoperitoneum from the closed technique to the open technique to increase patient safety. The rate of complications related to needle and/or trocar insertion subsequently decreased as the surgeon's experience performing laparoscopic surgery using the open-entry technique increased [13].

Our study also consists of a selected series of patients, because $51 \%$ of them had a history of surgery and only $46 \%$ had a normal BMI $\left(20-25 \mathrm{~kg} / \mathrm{m}^{2}\right)$. It is well recognized that the introduction of pneumoperitoneum and trocars in obese patients is difficult because of the lack of feeling the instruments penetrate the fascia or the insertion is too deep. However, most patients at risk are lean. In these patients the distance between the abdominal wall and the underlying structures is short so that a Veress needle or trocar penetrating the abdominal fascia and peritoneum with a little too much force puts these structures at risk. Furthermore, the risk of bowel injury is increased in patients who had previous abdominal surgery because of adhesions of the small bowel to the abdominal wall. Nevertheless, in the present series of patients a closed-entry technique was used despite the increased risk of complications caused by an abnormal BMI and the risk of adhesions after previous surgery. It is remarkable that in only one third of the claims informed consent was properly given and documented, and in only $48 \%$, were complications discussed with the patient preoperatively. An unexpected negative outcome that is neither discussed preoperatively nor explained postoperatively is probably the most important trigger for litigation. A properly informed patient is less likely to file a claim [20]. This group of patients consisted of young patients who underwent routine nonadvanced surgical procedures and both surgeon and patient did not expect such a serious complication. Another factor that may have provoked litigation is that more than half of the entry-related complications were diagnosed with a delay and that several of these patients had already been discharged. Therefore, it is important to discuss with the patient the risk of conversion to open surgery and the risk of vascular and bowel injuries in general, and to point out that not all of these injuries are diagnosed immediately. This conversation, including the informed consent of the patient, must be documented and patients at risk (e.g., lean or obese patients or those who had previous abdominal surgery) should be identified. Furthermore, it is important to keep in mind that entry-related complications still occur at a constant rate, even in routine procedures.

This study has several limitations. First, it probably represents only a part of all entry-related complications that occur. Furthermore, the study consists of a small, retrospectively collected, and selected population of patients, probably representing the most dramatic cases.
During the study period a (growing) fraction of the hospitals in the Netherlands were insured at MediRisk. Because of insufficient record-keeping, we were unable to clearly identify risk factors or specific trocar devices at risk. In general, this study does not present any data on the total population who had a laparoscopic procedure by the same surgeons responsible for the claims analyzed. Therefore, the definite relationship between several factors associated with entry-related injuries could no be established.

In conclusion, entry-related complications provoking litigation probably comprise one fifth of all laparoscopy-related claims. Patients that filed a claim were mostly young females with a history of abdominal surgery who were operated on in a day-care setting or had short-stay surgery with severe consequences of the entry-related complications. Most claims involved the closed-entry technique.

\section{References}

1. Jansen FW, Kolkman W, Bakkum EA, de Kroon CD, TrimbosKemper TC, Trimbos JB (2004) Complications of laparoscopy: an inquiry about closed- versus open-entry technique. Am J Obstet Gynecol 190(3): 634-638

2. Bonjer HJ, Hazebroek EJ, Kazemier G, Giuffrida MC, Meijer WS, Lange JF (1997) Open versus closed establishment of pneumoperitoneum in laparoscopic surgery. Br J Surg 84(5): 599-602

3. Philips PA, Amaral JF (2001) Abdominal access complications in laparoscopic surgery. J Am Coll Surg 192(4): 525-536

4. Bhoyrul S, Vierra MA, Nezhat CR, Krummel TM, Way LW (2001) Trocar injuries in laparoscopic surgery. J Am Coll Surg 192(6): 677-683

5. Fuller J, Ashar BS, Carey-Corrado J (2005) Trocar-associated injuries and fatalities: an analysis of 1399 reports to the FDA. J Minim Invasive Gynecol 12(4): 302-307

6. Soderstrom RM (1993) Bowel injury litigation after laparoscopy. J Am Assoc Gynecol Laparosc 1(1): 74-77

7. Corson SL, Chandler JG, Way LW (2001) Survey of laparoscopic entry injuries provoking litigation. J Am Assoc Gynecol Laparosc 8(3): 341-347

8. van der Voort M, Heijnsdijk EA, Gouma DJ (2004) Bowel injury as a complication of laparoscopy. Br J Surg 91(10): 1253-1258

9. Neudecker J, Sauerland S, Neugebauer E, Bergamaschi R, Bonjer HJ, Cuschieri A, et al. (2002) The European Association for Endoscopic Surgery clinical practice guideline on the pneumoperitoneum for laparoscopic surgery. Surg Endosc 16(7): 11211143

10. Jansen FW, Kapiteyn K, Trimbos-Kemper T, Hermans J, Trimbos JB (1997) Complications of laparoscopy: a prospective multicentre observational study. Br J Obstet Gynaecol 104(5): 595-600

11. Harkki-Siren P, Kurki T (1997) A nationwide analysis of laparoscopic complications. Obstet Gynecol 89(1): 108-112

12. Hasson HM (1971) A modified instrument and method for laparoscopy. Am J Obstet Gynecol 110(6): 886-887

13. Hashizume M, Sugimachi K (1997) Needle and trocar injury during laparoscopic surgery in Japan. Surg Endosc 11(12): 11981201

14. Neudecker J, Sauerland S, Lefering R, Neugebauer E (2001) Closed versus open approach for laparoscopic surgery (Protocol). Cochrane Database Syst Rev (3): CD003547; DOI: 10.1002/ 14651858.CD003547

15. Medeiros LR, Fachel JM, Garry R, Stein AT, Furness S (2005) Laparoscopy versus laparotomy for benign ovarian tumours. Cochrane Database Syst Rev (3): CD004751

16. Schwenk W, Haase O, Neudecker J, Muller JM (2005) Short term benefits for laparoscopic colorectal resection. Cochrane Database Syst Rev (3): CD003145 
17. Levinson CJ (1974) Laparoscopy is easy-except for the complications: a review with suggestions. J Reprod Med 13(5): 187194

18. Levy BS, Hulka JF, Peterson HB, Phillips JM (1994) Operative laparoscopy: American Association of Gynecologic Laparoscopists, 1993 membership survey. J Am Assoc Gynecol Laparosc 1(4 Pt 1): 301-305
19. Merlin TL, Hiller JE, Maddern GJ, Jamieson GG, Brown AR, Kolbe A (2003) Systematic review of the safety and effectiveness of methods used to establish pneumoperitoneum in laparoscopic surgery. Br J Surg 90(6): 668-679

20. Strasberg SM (2005) Biliary injury in laparoscopic surgery: part 2. Changing the culture of cholecystectomy. J Am Coll Surg 201(4): 604-611 